\title{
A Novel Cell-Based Therapy for Contusion Spinal Cord Injury Using GDNF-Delivering NIH3T3 Cells with Dual Reporter Genes Monitored by Molecular Imaging
}

\author{
Wen-Cheng Lo ${ }^{1}$, Chung-Huei Hsu ${ }^{2}$, Alexander T.H. Wu ${ }^{3}$, Liang-Yo Yang ${ }^{4,5}$, Wei-Hong Chen ${ }^{6}$, Wen-Ta Chiu ${ }^{7}$, \\ Wen-Fu Lai ${ }^{3}$, Chih-Hsiung $\mathrm{Wu}^{8}$, Juri G. Gelovani ${ }^{9}$, and Win-Ping Deng ${ }^{3}$ \\ ${ }^{1}$ Graduate Institute of Clinical Medicine, Taipei Medical University, Taipei, Taiwan; ${ }^{2}$ Department of Nuclear Medicine, Taipei Medical \\ University, Taipei, Taiwan; ${ }^{3}$ Graduate Institute of Biomedical Materials and Engineering and Stem Cell Center, Taipei Medical \\ University, Taipei, Taiwan; ${ }^{4}$ Department of Physiology, College of Medicine, Taipei Medical University, Taipei, Taiwan; ${ }^{5}$ Graduate \\ Institute of Neuroscience, College of Medicine, Taipei Medical University, Taipei, Taiwan; ${ }^{6}$ Graduate Institute of Medical Sciences, \\ Taipei Medical University, Taipei, Taiwan; ${ }^{7}$ Taipei Municipal Wan Fang Hospital and Taipei Medical University, Taipei, Taiwan; \\ ${ }^{8}$ Division of General Surgery, Department of Surgery and Cancer Center, Taipei Medical University Hospital, Taipei, Taiwan; and \\ ${ }^{9}$ Experimental Diagnostic Imaging, M.D. Anderson Cancer Center, Houston, Texas
}

\begin{abstract}
This aim of our study was to evaluate a novel cell-based therapy for contusion spinal cord injury (SCl) using embryonic-derived NIH3T3 cells, which endogenously express glial cell line-derived neurotrophic factor (GDNF). Methods: Proliferation and differentiation of transplanted NIH3T3 cells and their anti-apoptotic effects were examined after their engraftment into the spinal cords of Long-Evans rats subjected to acute SCI at the T10 vertebral level by a New York University impactor device. NIH3T3 cells were initially engineered to contain dual reporter genes, namely thymidine kinase $(T)$ and enhanced green fluorescence protein $(\mathrm{G})$, for in vivo cell tracking by both nuclear and fluorescence imaging modalities. Results: Planar and fluorescence imaging demonstrated that transplanted NIH3T3-TG cells at the L1 vertebral level migrated $2 \mathrm{~cm}$ distal to the injury site as early as $2 \mathrm{~h}$, and the signals persisted for $48 \mathrm{~h}$ after SCI. The expression of GDNF by NIH3T3-TG cells was then confirmed by immunohistochemical analysis both in vitro and in vivo. GDNFsecreting NIH3T3-TG transplant provided anti-apoptotic effects in the injured cord over the period of 3 wk. Finally, NIH3T3-TG cells cultured under neuronal differentiation medium exhibited both morphologic and genetic resemblance to neuronal cells. Conclusion: GDNF-secreting NIH3T3-TG cells in combination with molecular imaging could be a platform for developing therapeutic tools for acute SCI.
\end{abstract}

Key Words: molecular imaging; neurology; animal imaging; GDNF; GFP; spinal cord injury

J Nucl Med 2008; 49:1512-1519

DOI: 10.2967/jnumed.108.051896

\footnotetext{
Received Feb. 23, 2008; revision accepted May 20, 2008.

For correspondence or reprints contact: Win-Ping Deng, Institute of Biomedical Materials and Engineering and Stem Cell Center, Taipei Medical University, 250 Wu-Xing St., Taipei, Taiwan 110.

E-mail: wpdeng@ms41.hinet.net

COPYRIGHT @ 2008 by the Society of Nuclear Medicine, Inc.
}

D espite extensive research, clinical advancements, and improved rehabilitation strategies, spinal cord injury (SCI) remains a significant cause of disability and mortality, with an estimated 2.5 million people living with this injury (1). SCI, in general, comprises 2 phases. The first phase is the initial physical insult to the spinal cord, which can be caused by various types and extents of trauma, leading to contusion, compression, penetration, or maceration of the cord. SCI leads to the death of neurons (supporting cells including oligodendrocytes, astrocytes, and precursor cells) and to cavities and cysts, which may disrupt the ascending or descending axonal tracts. The second phase consists of destructive cellular events including progressive degeneration in the initially intact axons by ischemia, inflammation, free radical-induced cell death, glutamate excitotoxicity, cytoskeletal degradation, and induction of extrinsic and intrinsic apoptotic pathways (2). These secondary pathologic states represent an important target in developing protective strategies for treatment of SCI.

Strategies have been used to focus on the prevention of further neuronal death or to induce the regeneration of these cells in SCI animal models. Exogenous administration of growth factors that modulate neuronal survival, neurite outgrowth, synaptic plasticity, and neurotransmission has been proposed as a potential therapeutic treatment for SCI. For instance, glial cell line-derived neurotrophic factor (GDNF) has been shown to have a potent survival-promoting effect on various neuronal populations, including dopaminergic (3), noradrenergic (4), cortical (5), retinal ganglion (6), sensory $(7,8)$, and motor neurons (9). In addition, GDNF induced the growth of motor and sensory axons and remyelination in laboratory rats with partial and complete 
spinal cord transections $(10,11)$. Another promising strategy in SCI repair being developed is stem cell- or progenitor cell-based therapy. It is hoped that these undifferentiated cells will provide an inexhaustible source of neurons and glia for therapies aimed at cell replacement or neuroprotection in disorders affecting the central nervous system. The transplantation of stem cells could promote functional recovery by reconstituting damaged nerve tracts, remyelinating axons, and increasing plasticity or axon regeneration (12). For instance, locomotor improvements have been demonstrated in spinal cord-injured mice and primates (13-15). However, no single therapeutic methodology has been proven effective enough for significant functional recovery because of difficulties such as posttransplantation cellular lineage restriction (16), variable host responses to the engraftment $(17,18)$, and unfavorable hostile environment for the implanted cells.

To develop a novel therapy consisting of GDNF-secreting cells for the amelioration of SCI using a rat model, we used gene-modified NIH3T3 cells (NIH3T3-TG), which contain dual reporters, namely herpes simplex virus type I thymidine kinase (HSV1-TK; T) and Aequorea victoria-enhanced green fluorescent protein (eGFP; G), under control of 2 separate promoters (19). NIH3T3-TG cells were engineered to provide 2 purposes. First, NIH3T3-TG cells represent an ideal GDNF-delivery vessel because they are embryoderived fibroblasts that naturally secrete a substantial amount of GDNF (20) and maintain a stem cell-like differentiation flexibility (21). Second, the dual reporter genes, TG, provide means for both in vitro and in vivo tracking of these cells and markers for cellular incorporation and differentiation on transplantation. The results showed that genetically modified NIH3T3-TG cells enabled tracking of their migration and differentiation in the wake of transplantation up to $3 \mathrm{wk}$. In addition, grafted NIH3T3TG cells were able to secrete GDNF in vivo and provided anti-apoptotic effects to the injured cord. Furthermore, NIH3T3-TG cells appeared to take on neuronal-like morphology and reacted positively against anti-glial fibrillary acidic protein (GFAP). This unique cellular therapeutic intervention, combining the neuronal-protection offered by GDNF, differentiation flexibility of NIH3T3 cells, and stable reporter genes, could provide the foundation for developing clinical treatment for SCI in the future.

\section{MATERIALS AND METHODS}

\section{Cell Culture and In Vitro Neuronal Induction}

Embryo-derived NIH3T3-TG cells, genetically modified to contain dual reporter genes (TK and eGFP), were a generous gift from Dr. W. K. Yang, Department of Biomedical Sciences, Chung Shan Medical University, and were cultured in Dulbecco's modified Eagle's medium (DMEM) with $10 \%$ calf serum. Neuronal differentiation was performed as described previously (22). Briefly, NIH3T3-TG cells were seeded at a density of 500 cells $/ \mathrm{cm}^{2}$ and maintained in $\alpha$-modified minimum essential medium and $20 \%$ fetal bovine serum. Twenty-four hours before neuronal induction, me- dium was replaced with preinduction medium consisting of DMEM, $20 \%$ fetal bovine serum, $1 \mathrm{mM} \beta$-mercaptoethanol, and recombinant human fibroblast growth factor-2 (rhFGF-2; PeproTech, Inc.) $(5 \mathrm{ng} / \mathrm{mL}$ ). For neuronal induction, the preincubation medium was removed and the cells were washed with phosphate-buffered saline (PBS) followed by a $5 \mathrm{~h}$-incubation period in neuronal induction medium composed of DMEM and $10 \mathrm{mM} \beta$-mercaptoethanol. Cells were then fixed using $80 \%$ ice-cold methanol for immunohistochemical analysis as described in a later section.

\section{RNA Extraction and Semiquantitative Polymerase Chain Reaction (PCR) Analysis}

Total RNA harvested from subconfluent monolayer cultures was extracted using reagent (TRIzol; Invitrogen Life Technologies) and underwent reverse transcription (RT) followed by PCR amplification. Reverse transcription was performed with SuperScript III (Invitrogen Life Technologies) and an Oligo (dT) $)_{12-18}$ primer. Four micrograms of RNA were added into a final volume of $21-\mu \mathrm{L}$ solution containing $10 \mathrm{mM}$ deoxynucleotide triphosphate mix, $10 \times$ RT buffer, $25 \mathrm{mM} \mathrm{MgCl}_{2}, 0.1 \mathrm{M}$ dithiothreitol, RNase inhibitor, and RNase H. Six micrograms of RT product were used for the PCR amplification in a final volume of $50 \mu \mathrm{L}$ containing $2.5 \mathrm{mM}$ deoxynucleotide triphosphate, $25 \mathrm{mM} \mathrm{MgCl}_{2}$, upstream or downstream primers (nestin and GDNF), and Taq DNA polymerase (Invitrogen Life Technologies). PCR amplification of reverse-transcribed cDNA was performed with the following primers and conditions. The primers used for nestin are 5'GTGCCTCTGGATGATG 3' (forward) and 5'TTGACCTTCCTCCCCCTC $3^{\prime}$ (reverse). Tm: $55^{\circ} \mathrm{C}, 30$ cycles. The primers for GDNF are 5'CCGGACGGGACTCTAAGATGAA 3' (forward) and 5' GTCAGATACATCCACACCGTTT 3' (reverse). Tm: 56 ${ }^{\circ} \mathrm{C}, 30$ cycles. PCR products were then run on $1 \%$ agarose gels (Agarose I; AMRESCO) and visualized with ethidium bromide staining. Images were analyzed using FloGel-I (Fluorescent Gel Image System; TOP BIO Co.). Glyceraldehyde 3-phosphate dehydrogenase was used as an internal control.

\section{Rat SCI Model}

A total of 20 female Long-Evans rats (200-220 g) were anesthetized with sodium pentobarbital $(40 \mathrm{mg} / \mathrm{kg}$, intraperitoneally), and a laminectomy was performed by exposing the dura matter at spinal cord level T10. The vertebral column was stabilized at T8 and T12. Spinal cord contusion was performed using the New York University impactor device (23) by dropping a 10 -g weight from a $2.5-\mathrm{cm}$ height onto the exposed cord. Control animals received the laminectomy alone. After surgery, rats were kept at $37^{\circ} \mathrm{C}$ and closely monitored. Urine was expressed manually twice per day, and rats were checked daily to monitor their overall health. All protocols were approved by the Taipei Medical University Animal Care and Use Committee.

\section{Cell Transplantation and Planar $\gamma$-Camera Imaging (PGCl)}

PGCI was performed as described previously (24). Briefly, NIH3T3TG cells were prelabeled with $185 \mathrm{kBq} / \mathrm{mL}$ of ${ }^{131}$ I-labeled $2^{\prime}$-fluoro- $2^{\prime}$ deoxy-5'-iodo-1 $\beta$-D-arabinofuranosyluracil ( ${ }^{131} \mathrm{I}$-FIAU) for $16 \mathrm{~h}$ and washed 3 times with PBS before transplantation. Labeled NIH3T3-TG cells $\left(1 \times 10^{6}\right.$ cells in $50 \mu \mathrm{L}$ of PBS $)$ were then directly injected into the spinal cords of the rat ( $25 \mu \mathrm{L}$ into each side of $\mathrm{L} 1$ level of the cord) using a microsyringe (700 series; Hamilton) immediately after induced SCI. For in vivo tracking of ${ }^{131}$ I-FIAU-labeled NIH3T3-TG cells, static images were collected at 2, 24, and $48 \mathrm{~h}$ after injection by a digital 
FIGURE 1. In vivo imaging of migration and targeting of ${ }^{131} \mathrm{I}-\mathrm{FIAU}$-labeled NIH3T3-TG, GDNF-delivering cells in rats. (A) Representative serial planar imaging of SCl-induced rats $(n=6)$ at 2,24 , and $48 \mathrm{~h}$ after transplantation of $1 \times 10^{6}$ NIH3T3-TG cells labeled with ${ }^{131}$ IFIAU. Labeled NIH3T3-TG cells could be detected at injury site as early as $2 \mathrm{~h}$ after transplantation. (B) Optical eGFP fluorescence imaging of same rat from 48-h planar imaging. Fluorescence imaging demonstrated migratory pattern of transplanted NIH3T3-TG cells similar to one observed by planar imaging. White line and asterisk represent injury site and transplantation site, respectively.

$\gamma$-camera (SP-6; Elscint), equipped with a high-energy pinhole collimator. Images were acquired at $100 \mathrm{~K}$ counts per frame at $2 \mathrm{~h}$ and after images were acquired by a preset time mode.

\section{Immunohistochemical Analysis}

Spinal cords were collected from sacrificed rats at different time points for histologic analysis. The spinal cords were fixed for $24 \mathrm{~h}$ in $4 \%$ paraformaldehyde in $0.14 \mathrm{M}$ Sorensen's phosphate buffer, $\mathrm{pH}$ 7.4. Tissue was washed 3 times and stored overnight in buffer. The tissue was placed in $30 \%$ sucrose solution in PBS until the tissue sank. Cryosections were cut, rinsed, and blocked with $10 \%$ rabbit serum and $0.4 \%$ Triton X-100 in Tris-buffered saline or PBS for $30 \mathrm{~min}$ at room temperature. Sections were incubated with respective goat anti-GDNF and anti-nestin antibodies (1:100; Santa Cruz Biotechnology Inc.) overnight at $4^{\circ} \mathrm{C}$. The sections were washed 3 times with Tris-buffered saline, 5 min each. After

A T10

L1
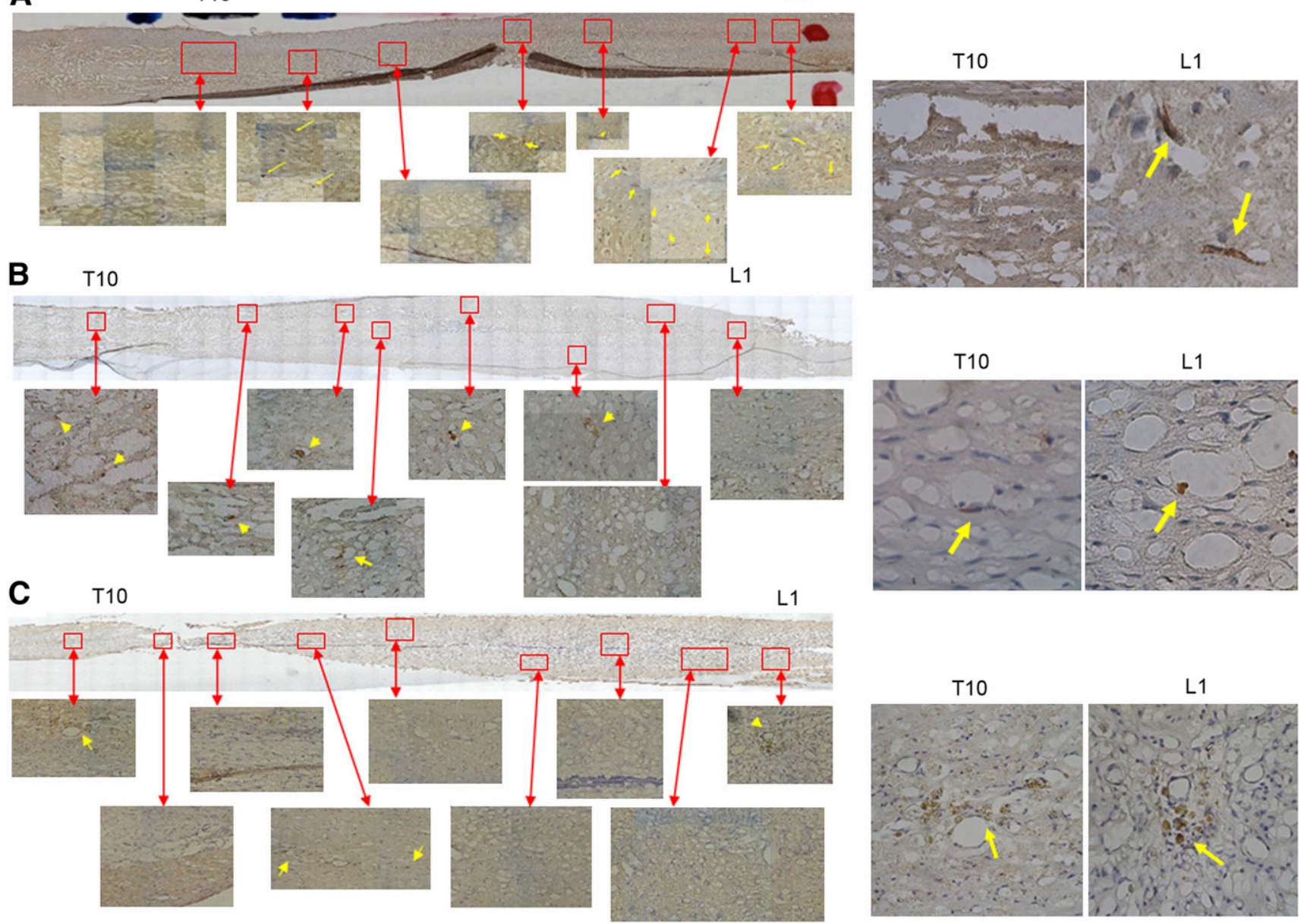

T10

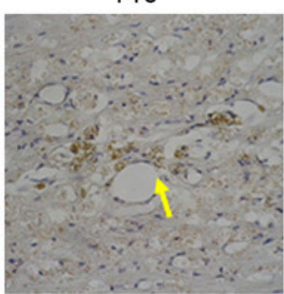

L1

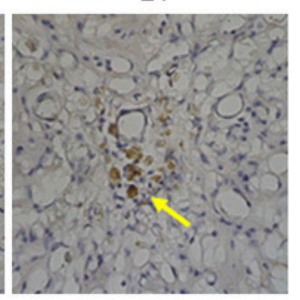

FIGURE 2. Serial histologic examination of transplanted NIH3T3-TG cells in rat contused spinal cord sections. Numerous eGFPpositive cells (arrows) were detected at site of injection (L1) initially at $2 \mathrm{~h}(\mathrm{~A}), 48 \mathrm{~h}(\mathrm{~B})$, and $3 \mathrm{wk}(\mathrm{C})$ after transplantation. Inserts in right panels showed morphology of NIH3T3-TG (arrows) in higher magnifications. 
the sections were washed, they were incubated with secondary antibody (Alexa Fluor 594 donkey antigoat secondary antibody [1:1,000]; Invitrogen) for $1 \mathrm{~h}$ at room temperature in the dark and mounted with fluorescent mounting medium (Vectorshield). Slides were examined on a research microscope (BX51; Olympus). For nonfluorescence microscopic analysis of spinal cord crosssections, biotin-labeled secondary antibody followed by AMCA avidin D (Vector Laboratories) was used for visualization.

\section{Terminal Deoxynucleotidyl Transferase-Mediated dUTP Nick-End Labeling (TUNEL) Assay}

TUNEL assay was performed using the DeadEnd Fluorometric TUNEL System (Promega) according to the manufacturer's protocol. Spinal cord sections around the injury epicenter were collected and processed as described in the previous section followed by the incubation with tetrarhodamine isothiocyanateconjugated mouse IgG (1:100; Sigma). Finally, labeled sections were covered with a cover slide with mounting medium and visualized by fluorescence microscopy.

\section{RESULTS}

\section{In Vivo Imaging of NIH3T3-TG-Cell-Targeted SCI}

Because NIH3T3-TG cells contained dual reporter systems (TK [T] and enhanced eGFP [G]), both PGCI- and fluorescence-based optical imaging techniques were used for in vivo cellular tracing. After ${ }^{131}$ I-FIAU-labeled NIH3T3TG cells were injected into the site $2 \mathrm{~cm}$ distal to the injury site (designated as the injection site), planar imaging was obtained at the site of SCI as early as $2 \mathrm{~h}$ after injury and continued at least for $48 \mathrm{~h}$ (Fig. 1A). Information on ${ }^{131} \mathrm{I}-$ FIAU uptake by NIH3T3-TG cells can be found in Supplemental Figure 1 (supplemental materials are available online only at http://jnm.snmjournals.org). Strong eGFP fluorescence $48 \mathrm{~h}$ after SCI (Fig. 1B) displayed a homing pattern of NIH3T3-TG cells similar to the one observed with planar imaging, indicating that the injected cells were responsive to and capable of homing from L1 (injection site) to T10 (injury site). As judged from the eGFP fluorescence signal, the majority of the injected NIH3T3-TG cells reached midway between the injury and injection sites.

\section{In Situ Validation of Survival and Engraftment of Tracer NIH3T3-TG Cells in Rat-Contused Spinal Cord}

To validate in vivo imaging results and to determine cellular incorporation, rats receiving NIH3T3-TG grafts were euthanized at $2 \mathrm{~h}, 48 \mathrm{~h}$, and $3 \mathrm{wk}$ after transplantation. Frozen spinal cord sections from T10 to L1 were stained
A

L1
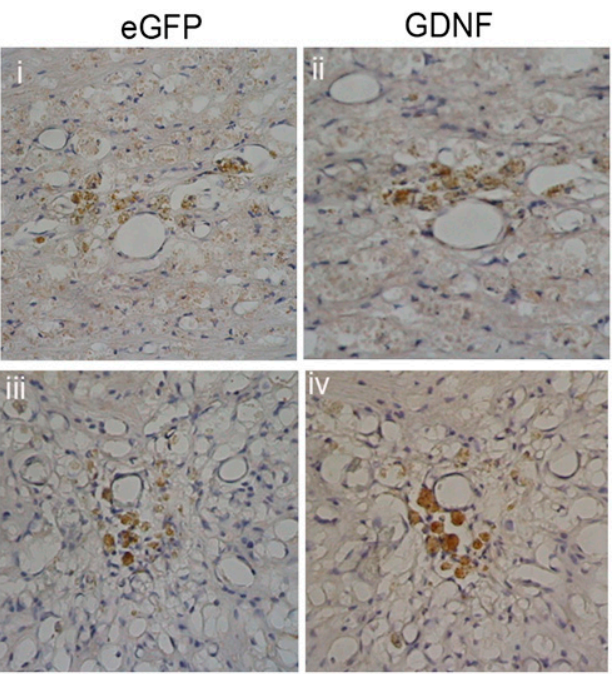

B
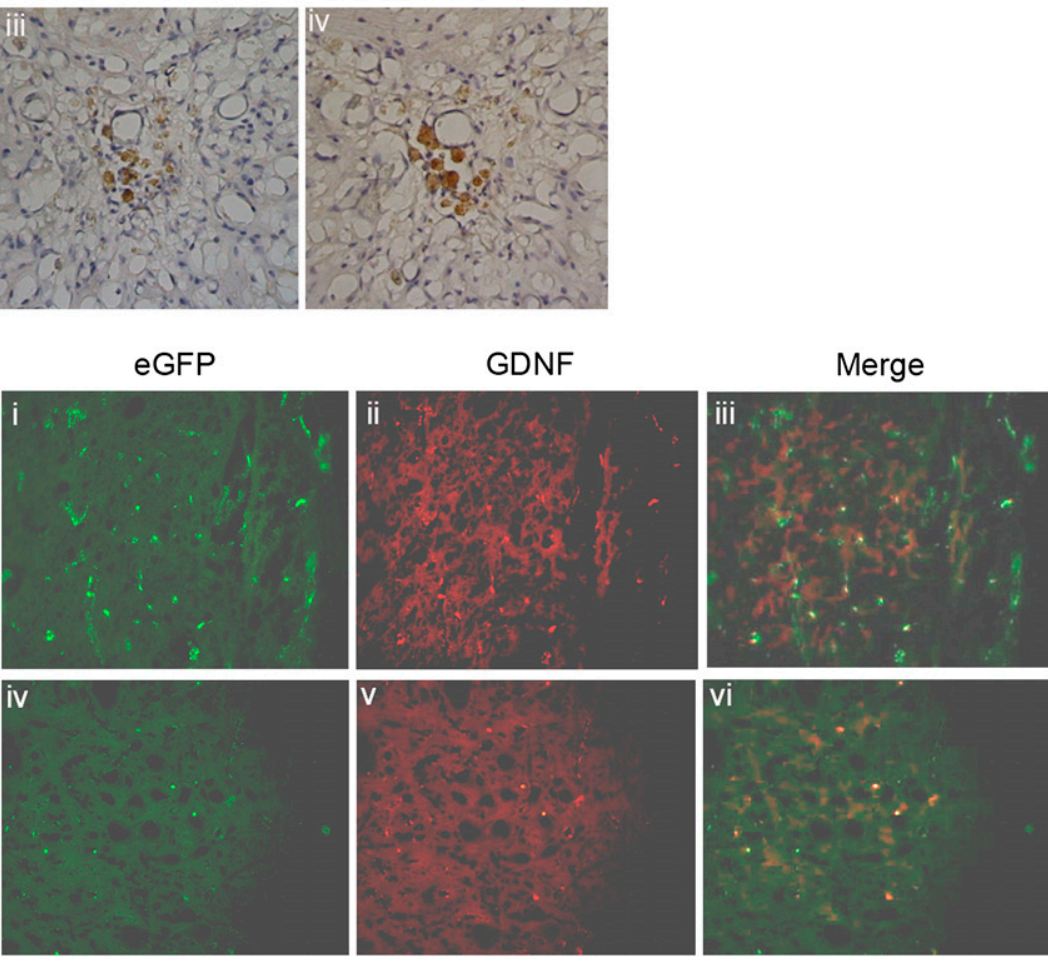

FIGURE 3. Immunohistologic examination of GDNF-expressing cells in SClinduced rat spinal cord. (A) Representative serial cross-section of injured cord $3 \mathrm{wk}$ after transplantation. Numerous eGFPcontaining cells revealed by immunoperoxidase staining were located mainly around injury epicenter (T10, i) and injection site (L1, iii). Adjacent tissue sections probed with anti-GDNF antibody also exhibited similar staining pattern (ii and iv, respectively). (B) Double immunofluorescence study for both anti-eGFP (i and iv) and anti-GDNF (ii and v) of injured cord 3 wk after transplantation. Cells positive for anti-eGFP and anti-GDNF antisera were detected at injury site (i and ii, respectively) and injection site (iv and $\mathrm{v}$, respectively). Double-labeling of eGFP and GDNF signals was demonstrated by merged images at both injury site (T10, iii) and injection site (L1, vi). Magnification 200x. 
with anti-eGFP antiserum for immunohistochemical analysis. Most eGFP-positive cells were observed at the site of injection initially (L1, Fig. 2A) and exhibited typical elongated NIH3T3-TG morphology (yellow arrows in Fig. 2A, insert). Subsequently NIH3T3-TG cells migrated rostally toward the injury site (T10, yellow arrows in Figs. 2B and $2 \mathrm{C}$ ) and were still detectable along the cord segments, T10 to L1, $48 \mathrm{~h}$ (Fig. 2B) and $3 \mathrm{wk}$ (Fig. 2C) after transplantation, in accordance with the in vivo imaging results. Migrating NIH3T3-TG cells were found mainly around the injured sites (Figs. $2 \mathrm{~B}$ and 2C, insert).

\section{Expression of GDNF by Transplanted NIH3T3-TG Cells in SCI-Induced Rat Spinal Cord}

To demonstrate that the transplanted NIH3T3-TG cells could act as a GDNF-delivering vehicle, immunohistochemical analysis of the spinal cord sections from both the injury (T10) and injection (L1) sites was conducted. Immunoperoxidase staining revealed that eGFP- and GDNFpositive cells were found at the sites of injury (Fig. 3A, $\mathrm{i}$ and ii) and injection (Fig. 3A, iii and iv). Double-labeling of immunofluorescence with both anti-eGFP and anti-GDNF antisera was performed to confirm that these GDNFexpressing cells were indeed NIH3T3-TG. In agreement with the immunoperoxidase staining results, at both injury and injection sites cells exhibiting both anti-eGFP (Fig. 3B, $\mathrm{i}$ and iv) and anti-GDNF (Fig. 3B, ii and v) signals were detected, confirming that engrafted NIH3T3-TG cells expressed GDNF (Fig. 3B, iii and vi).

\section{Anti-Apoptotic Effects by Transplanted GDNF-Secreting NIH3T3-TG Cells}

To investigate the anti-apoptotic effects of GDNF-expressing NIH3T3-TG cells on the injured spinal cord, the cells from both NIH3T3-TG-transplanted and -nontransplanted animals were evaluated by TUNEL assay on frozen sections obtained from the periphery of the injury epicenter (Fig. 4A). TUNEL assay revealed that the number of apoptotic cells (green, fluorescein isothiocyanate) in the spinal cords of SCI rats that received GDNF-expressing NIH3T3-TG transplants (Fig. 4B, vi-viii) were substantially fewer than their respective controls without transplants (Fig. 4B, ii-iv). Quantitatively, there were approximately 2.6-fold fewer apoptotic cells in NIH3T3-TG-transplanted cord sections (please see inserted table in Fig. 4A) than in the nontransplanted cords.

\section{Colocalization of GFAP-Positive and NIH3T3-TG Cells}

The anti-apoptotic effect observed in spinal cords of NIH3T3-TG-grafted rats was further validated by immunohistochemistry using spinal cord cross-sections from rats 3 wk after transplantation. At both the injury site (T10, Figs. 5A and 5B) and the injection site (L1, Figs. 5C and 5D), numerous immunopositive GFAP (arrowheads in Figs. 5A and 5C) and eGFP (arrowheads in Figs. 5B and 5D) cells were detected. Both GFAP- and eGFP-positive cells appeared to be colocalized or near each other, implicating the possibility of astrocytes derived from transplanted NIH3T3-TG cells.
FIGURE 4. Prevention of cellular death by GDNF-secreting NIH3T3-TG xenograft. (A) Schematic representation demonstrates collection of circumferential cross-sections of injured cord 3 wk after transplantation. (B) Comparative micrographs illustrating immunofluorescent TUNEL reaction for detection of cellular apoptosis surrounding lesion center of sham-injected (untreated group) (i-iv) and NIH3T3-TG-grafted (v-viii) rat spinal cords. Apoptosis (green) was more pronounced in untreated group (ii, iv), compared with NIH3T3-TG-grafted (vi, viii) cord sections. Nuclei were stained with propidium iodide (iii and vii). When quantified by in vivo TUNEL counts, NIH3T3TG-grafted spinal cord sections exhibited approximately 2.6-fold less cellular death, compared with untreated group. $\mathrm{PI}=$ propidium iodide; FITC = fluorescein isothiocyanate.
A
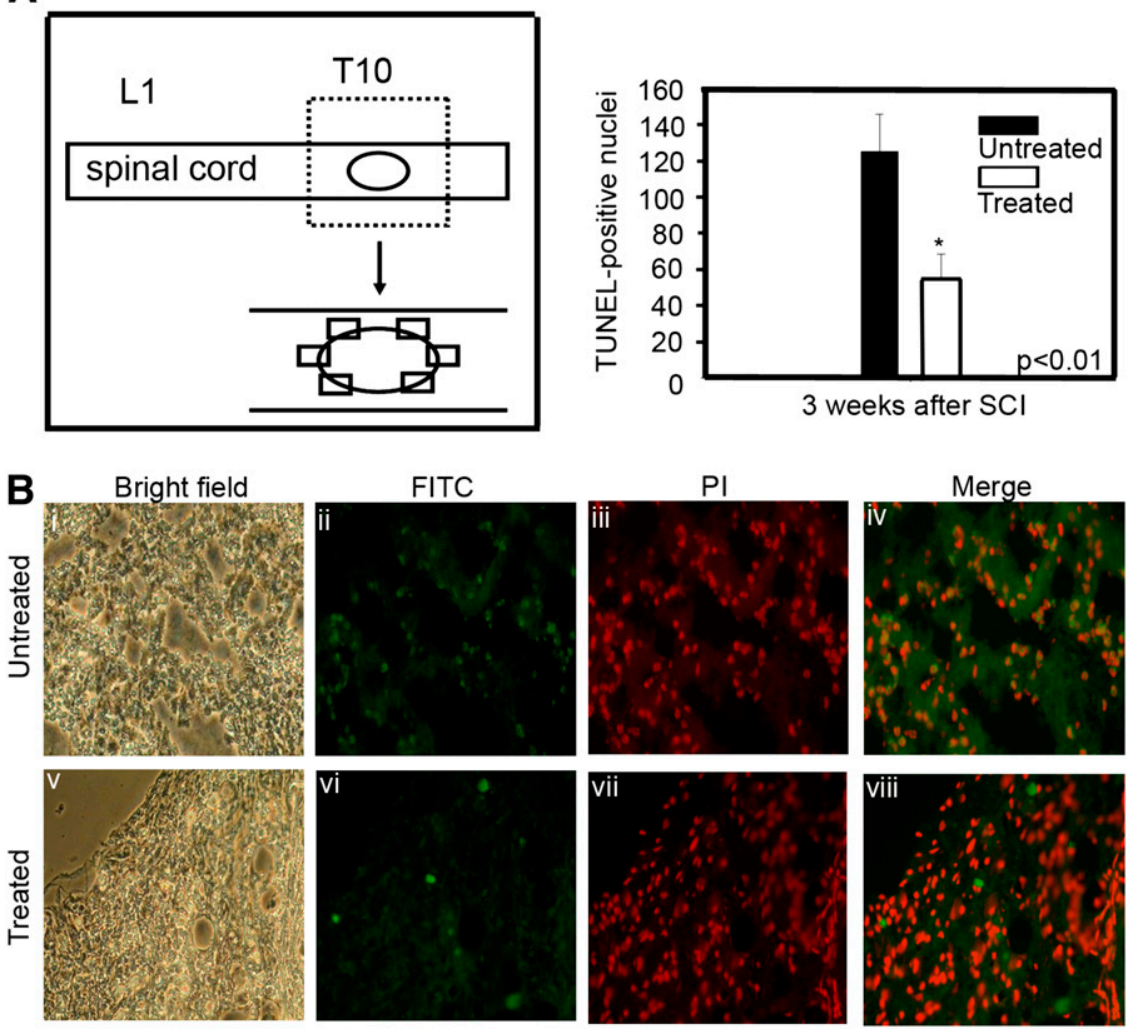

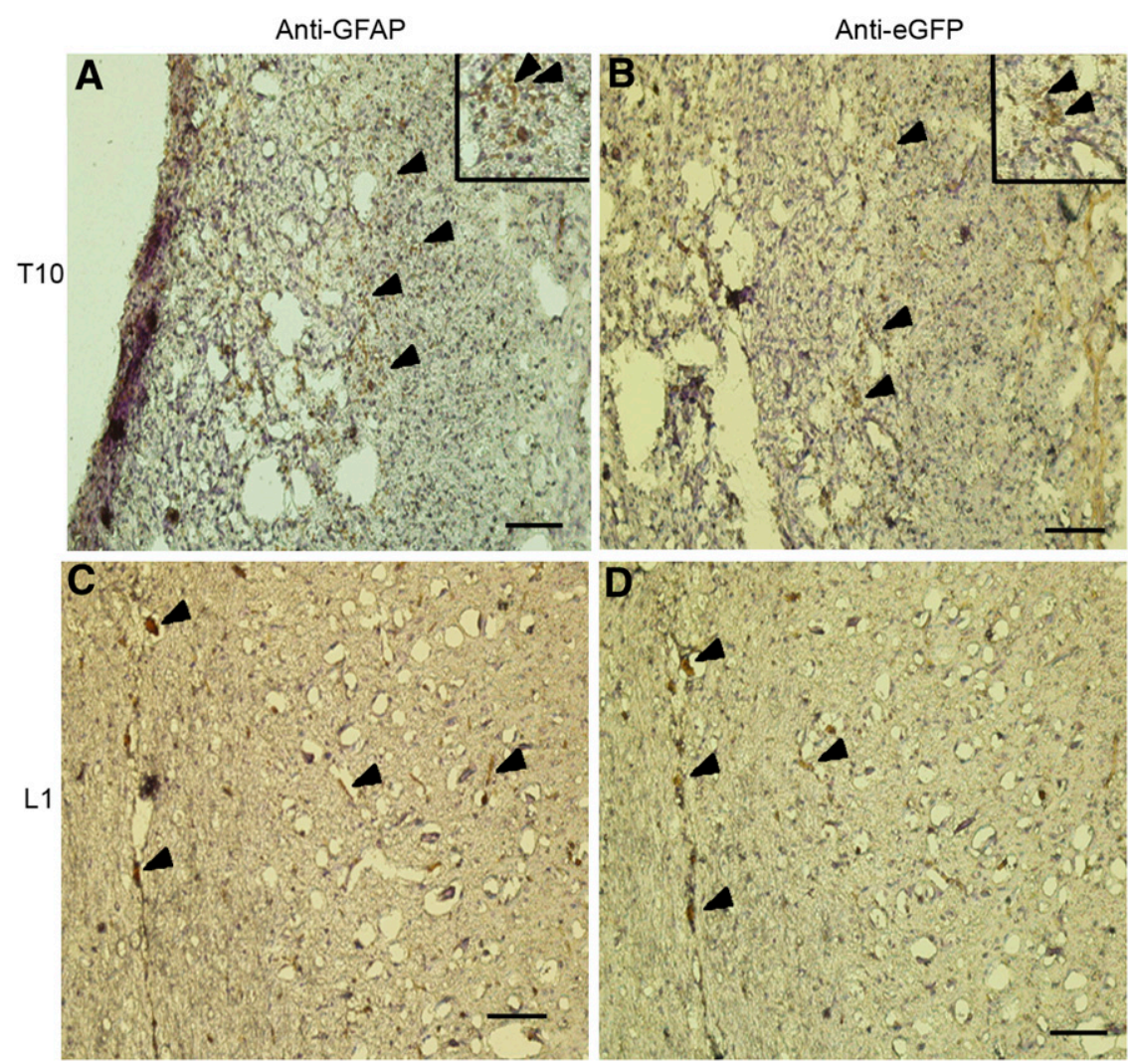

FIGURE 5. Colocalization of eGFP- and GFAP-positive cells in NIH3T3-TG-treated rat spinal cord. Serial spinal cord crosssections were probed with anti-eGFP and anti-GFAP antibodies. At injury epicenter (T10), numerous anti-GFAP (A) and antieGFP (B) cells were detected; anti-GFAP and anti-eGFP cells were detected at injection site ( $L 1, C$ and $D$, respectively) as well. Arrowheads show immunopositive cells. Inserts in A and B represent magnified images of stained areas. Scale bars $=$ $100 \mu \mathrm{m}$.

\section{In Vitro Neuronal Induction of NIH3T3-TG Cells}

After immunohistochemical analysis implicated the possible astrocytic differentiation of the transplanted NIH3T3TG cells in the injured rat spinal cords, we investigated whether NIH3T3-TG cells could be induced to neuronal cells in vitro. After neuronal induction, NIH3T3-TG cells (Fig. 6A, i) exhibited a neuronal-like morphology similar to that of rat neuronal PC-12 cells (Fig. 6A, ii). In addition, NIH3T3-TG cells were also immunopositive for the neuronal-epithelial marker nestin (Fig. 6B, i-iii). Furthermore, corroborating our in vivo data, these nestin-positive NIH3T3TG cells (Fig. 6C, i) also expressed GDNF (Fig. 6C, ii); GDNF was expressed at the transcriptional level (Fig. 6C, insert) as well, indicating that NIH3T3-TG could potentially be induced to be neuronal or glial cell lineages.

\section{DISCUSSION}

Our aim in this study was to develop a cellular vessel that contains reporter genes for in vivo tracking or identification of GDNF and delivers GDNF to the site of injury to prevent secondary neuronal death in the wake of acute SCI.

The accuracy of transplantation is a fundamental premise for cell-based therapy, particularly in small animals. Here we used dual-labeled NIH3T3-TG cells as a model. NIH3T3-TG cells were detectable in vivo by both PGCI and immunofluorescence imaging up to $2 \mathrm{~d}$. These cells appeared to be able to home to the injury epicenter $2 \mathrm{~cm}$ above the transplantation site as early as $2 \mathrm{~h}$ after transplantation as shown by the PGCI technique. A similar migratory pattern was also obtained in the anti-eGFP-probed immunohistochemical analysis of the cord sections. In this study, we used planar $\gamma$-camera imaging for the detection of NIH3T3-TG xenograft in SCI rats. This system could be improved in the future for the potential clinical application of SPECT (25) in acute SCI on transplantation to verify cell viability and homing pattern. A second reporter gene, eGFP, is also available in NIH3T3-TG cells for immunofluorescence imaging, thereby constituting a dual-modal imaging system. eGFP was used for both in vivo and in vitro tracking of the grafted NIH3T3-TG cells as demonstrated by immunofluorescence imaging and immunohistochemical analysis. Therefore, the homing ability of transplanted NIH3T3-TG can be clearly demonstrated by our dual-modality molecular imaging system. This system could provide a platform for future cellbased treatments in SCI.

Growth factors such as nerve growth factor, brain-derived neurotrophic factor, neurotrophin-3, ciliary neurotrophic factor, and GDNF all have been used to study their beneficial effects in spinal cord-injured animals (26). In this study, GDNF, a member of the transforming growth factor$\beta$ superfamily, was chosen as the candidate growth factor. GDNF exerts its function via a multicomponent receptor system including GDNF receptor $\alpha-1$ for ligand-receptor binding and rearranged-during-transfection protein for signal transduction. Many neurons with projections to the 


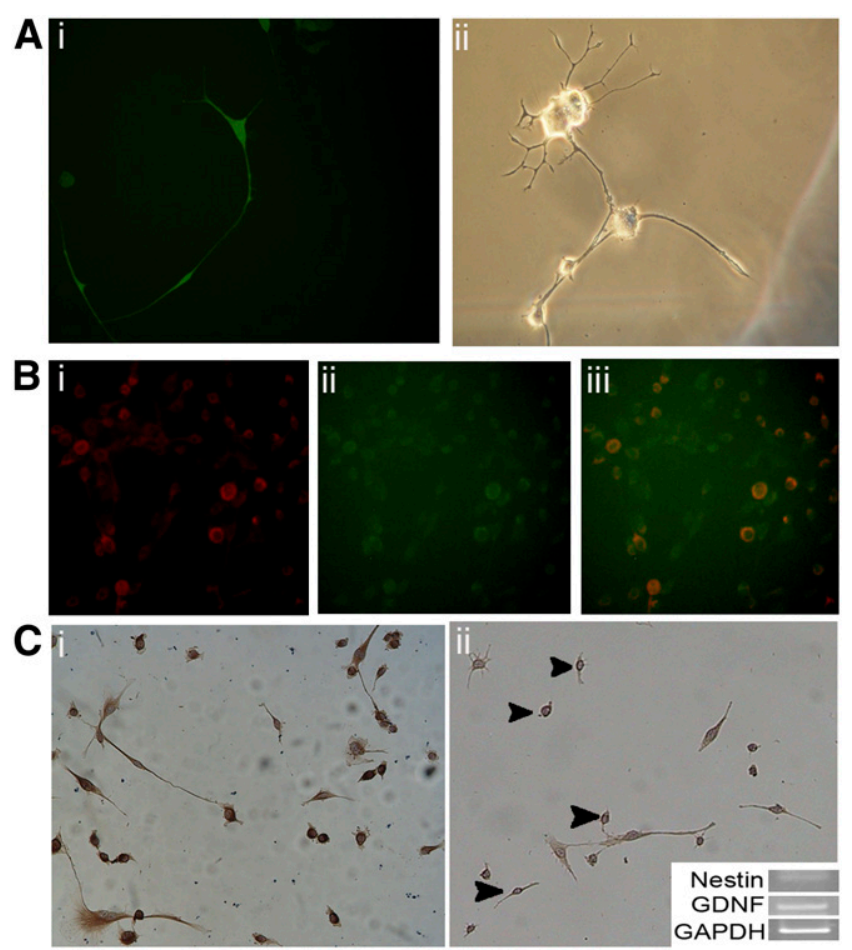

FIGURE 6. In vitro demonstration of neuronal differentiation potential of NIH3T3-TG cells. (A) NIH3T3-TG cells subjected to neuronal differentiation medium demonstrated elongated and branched morphology, resembling early neuronal cells (i, under fluorescence microscope, eGFP), compared with rat PC12 cells (ii, phase contrast image). (B) Immunofluorescence microscopic analysis of NIH3T3-TG cells after neuronal differentiation. NIH3T3-TG cells (green fluorescence, ii) after neuronal differentiation were both anti-nestin-positive (red fluorescence, i). (C) In vitro gene analysis of differentiated NIH3T3-TG cells. Neuronal-induced NIH3T3-TG cells were immunopositive in vitro for both nestin (i) and GDNF (ii) as well as immunopositive at transcriptional level (insert).

spinal cord are shown to express receptors for GDNF and its ligand; GDNF is upregulated after peripheral nerve injury (27) to prevent motor neuron death during development and adulthood $(28,29)$. On the basis of these findings, GDNF appears to be an ideal candidate for devising a therapeutic tool for the amelioration of SCI. Primary cells, particularly of neuronal lineages, have been proven difficult to maintain and expand in vitro; gene-manipulation procedures such as implementing reporter genes or therapeutic genes face challenges. An alternative cell source for exogenous GDNF delivery is required. NIH3T3 represents an ideal alternative. First, they are a well-established mouse embryo-derived fibroblast cell line used in a wide spectrum of gene-manipulation experiments. Reporter genes can be introduced into them with high efficiency. Additionally, NIH3T3 cells secrete GDNF endogenously (20) and NIH3T3 cells on appropriate stimuli in vitro or in vivo could differentiate into osteoblastslike cell lineage (30), indicating that these cells retain cell differentiation flexibility. Our data indicated that grafted NIH3T3-TG persisted and secreted GDNF in the injured spinal cord 3 wk after transplantation. An immediate upregulation of GDNF transcript after SCI followed by an increase in its protein expression in microglia and macrophages $1 \mathrm{~d}$ after injury have been reported (31), suggesting that endogenous GDNF could provide neuronal protection after the insult. GDNF detected $3 \mathrm{wk}$ after transplantation was most likely secreted by the engrafted NIH3T3-TG cells. More important, GDNF secreted by NIH3T3-TG cells appeared to be functional. This notion is supported by the in vivo comparative TUNEL staining of the NIH3T3-TG-treated and control spinal cord sections. The extent of apoptosis was significantly less in the NIH3T3-TG-grafted spinal cord, compared with the control. The anti-apoptotic effect exerted by and sustained GDNF secretion by NIH3T3-TG engraftment should provide a more permissive growth microenvironment for neuron regeneration or remyelination.

Because of the embryonic origin of NIH3T3-TG and its suggested differentiation flexibility, the possibility of differentiation into neuronal lineage was examined. Under neuronal differentiation medium, NIH3T3-TG cells exhibited a neuronal-like morphology similar to that of the rat pheochromocytoma cell line, PC12. The early neuroepithelial marker nestin was detected at both the transcriptional and the translational levels in vitro. In addition, both anti-eGFP- and anti-GFAP-positive NIH3T3-TG cells were found in the spinal cord sections $3 \mathrm{wk}$ after transplantation. These findings implicate that NIH3T3-TG cells in the injury epicenter could possibly differentiate into astrocytelike lineage. Our findings implicate that GDNF secreted by transplanted NIH3T3-TG cells might provide a more accommodating cellular environment for neural regeneration; thus, transplanted NIH3T3-TG cells were allowed to be in vivo educated and differentiated into astrocytelike cells. This improved cellular environment supplemented by GDNF-secreting NIH3T3-TG transplant after SCI meets our goal for the improvement of acute SCI.

Tumorigenic tendency for grafted cells has always been one of the concerns for stem cell-based therapy. NIH3T3TG-transplanted SCI rats did not exhibit abnormal tissue growth over the course of experiment as demonstrated by the spinal cord sections as well as macroscopic examination. This possibility has been taken into consideration when the reporter genes were inserted into NIH3T3 cells. One of the 2 reporter genes, thymidine kinase, can be used not only for molecular imaging purposes but also for self-destruction. If the transplanted NIH3T3-TG cells ever become tumorigenic, a pro-drug, ganciclovir, can be administered to induce thymidine kinase-mediated cell suicide (19).

\section{CONCLUSION}

PGCI and in vivo fluorescence imaging techniques revealed the homing of NIH3T3-TG cells to the injury epicenter on transplantation. In addition, NIH3T3-TG cells persisted in vivo $3 \mathrm{wk}$ after transplantation and demonstrated 
their ability to secrete GDNF in vivo. Furthermore, the transplanted NIH3T3-TG cells prevented secondary cell death in the cord. Finally, cultured NIH3T3-TG in neuronal differentiation medium exhibited a neuronal-like phenotype coined with the upregulation of nestin mRNA level and reacted positively with anti-GFAP antibody in the spinal cord cross-section. These findings indicate that NIH3T3TG treatment combines the advantage of the neuronalprotective effects of GDNF and the potential stem cell-like differentiation capacity of NIH3T3-TG cells, which could be valuable tools for developing a novel treatment for SCI or neuronal disorders.

\section{ACKNOWLEDGMENTS}

We thank Dr. Chung-Yi Hsu, the director of Topnotch Stroke Center, Taipei Medical University, Taipei, Taiwan, for critical reading of this manuscript and Dr. W.K. Yang, from the Department of Biomedical Sciences, Chung Shan Medical University, Taiwan, for his generous gift of the NIH3T3-TG cell line. We also thank Shiau-Jun Chen and Hon-Jian Wei from the Institute of Biomedical Materials and Engineering, Taipei Medical University, for their excellent technical assistance. This study is supported by Department of Health (DOH) DOH96-TD-G-111-013, Cancer Center and Stem Cell Center, Taipei Medical University Hospital, Taipei, Taiwan.

\section{REFERENCES}

1. Fehlings MG, Sekhon LH, Tator C. The role and timing of decompression in acute spinal cord injury: what do we know? What should we do? Spine. 2001; 26(suppl 24):S101-S110.

2. Crowe MJ, Bresnahan JC, Shuman SL, Masters JN, Beattie MS. Apoptosis and delayed degeneration after spinal cord injury in rats and monkeys. Nat Med. 1997;3:73-76

3. Lin LF, Doherty DH, Lile JD, Bektesh S, Collins F. GDNF: a glial cell linederived neurotrophic factor for midbrain dopaminergic neurons. Science. 1993; 260:1130-1132.

4. Arenas E, Trupp M, Akerud P, Ibanez CF. GDNF prevents degeneration and promotes the phenotype of brain noradrenergic neurons in vivo. Neuron. 1995; 15:1465-1473.

5. Wang Y, Lin SZ, Chiou AL, Williams LR, Hoffer BJ. Glial cell line-derived neurotrophic factor protects against ischemia-induced injury in the cerebral cortex. J Neurosci. 1997; 17:4341-4348.

6. Klocker N, Braunling F, Isenmann S, Bahr M. In vivo neurotrophic effects of GDNF on axotomized retinal ganglion cells. Neuroreport. 1997;8:3439-3442.

7. Molliver DC, Wright DE, Leitner ML, et al. IB4-binding DRG neurons switch from NGF to GDNF dependence in early postnatal life. Neuron. 1997;19: 849-861.

8. Bennett DL, Michael GJ, Ramachandran N, et al. A distinct subgroup of small DRG cells express GDNF receptor components and GDNF is protective for these neurons after nerve injury. J Neurosci. 1998;18:3059-3072.
9. Henderson CE, Phillips HS, Pollock RA, et al. GDNF: a potent survival factor for motoneurons present in peripheral nerve and muscle. Science. 1994;266: 1062-1064.

10. Blesch A, Tuszynski MH. GDNF gene delivery to injured adult CNS motor neurons promotes axonal growth, expression of the trophic neuropeptide CGRP, and cellular protection. J Comp Neurol. 2001;436:399-410.

11. Zhou L, Shine HD. Neurotrophic factors expressed in both cortex and spinal cord induce axonal plasticity after spinal cord injury. J Neurosci Res. 2003;74: 221-226.

12. Moraleda JM, Blanquer M, Bleda P, et al. Adult stem cell therapy: dream or reality? Transpl Immunol. 2006;17:74-77.

13. Cummings BJ, Uchida N, Tamaki SJ, et al. Human neural stem cells differentiate and promote locomotor recovery in spinal cord-injured mice. Proc Natl Acad Sci USA. 2005;102:14069-14074.

14. Iwanami A, Kaneko S, Nakamura M, et al. Transplantation of human neural stem cells for spinal cord injury in primates. J Neurosci Res. 2005;80:182-190.

15. Watanabe K, Nakamura M, Iwanami A, et al. Comparison between fetal spinalcord- and forebrain-derived neural stem/progenitor cells as a source of transplantation for spinal cord injury. Dev Neurosci. 2004;26:275-287.

16. Reier PJ. Cellular transplantation strategies for spinal cord injury and translational neurobiology. NeuroRx. 2004;1:424-451.

17. Ourednik J, Ourednik V. Graft-induced plasticity in the mammalian host CNS. Cell Transplant. 2004;13:307-318.

18. Ourednik V, Ourednik J. Multifaceted dialogue between graft and host in neurotransplantation. J Neurosci Res. 2004;76:193-204.

19. Hung SC, Deng WP, Yang WK, et al. Mesenchymal stem cell targeting of microscopic tumors and tumor stroma development monitored by noninvasive in vivo positron emission tomography imaging. Clin Cancer Res. 2005;11:77497756.

20. Ma D, Wang X, Han J. NIH 3 T3 cells or engineered NIH 3 T3 cells stably expressing GDNF can protect primary dopaminergic neurons. Neurol Res. 2000; 22:538-544.

21. Rubin H. 'Spontaneous' transformation as aberrant epigenesis. Differentiation. 1993;53:123-137.

22. Tsai MS, Hwang SM, Tsai YL, Cheng FC, Lee JL, Chang YJ. Clonal amniotic fluid-derived stem cells express characteristics of both mesenchymal and neural stem cells. Biol Reprod. 2006;74:545-551.

23. Basso DM, Beattie MS, Bresnahan JC. Graded histological and locomotor outcomes after spinal cord contusion using the NYU weight-drop device versus transection. Exp Neurol. 1996;139:244-256.

24. Deng WP, Wu CC, Lee CC, et al. Serial in vivo imaging of the lung metastases model and gene therapy using HSV1-tk and ganciclovir. $J$ Nucl Med. 2006;47: 877-884.

25. Ray $\mathrm{P}$, Bauer E, Iyer $\mathrm{M}$, et al. Monitoring gene therapy with reporter gene imaging. Semin Nucl Med. 2001;31:312-320.

26. Lu P, Tuszynski MH. Growth factors and combinatorial therapies for CNS regeneration. Exp Neurol. 2008;209:313-320.

27. Hammarberg H, Piehl F, Cullheim S, Fjell J, Hokfelt T, Fried K. GDNF mRNA in Schwann cells and DRG satellite cells after chronic sciatic nerve injury. Neuroreport. 1996;7:857-860.

28. Houenou LJ, Oppenheim RW, Li L, Lo AC, Prevette D. Regulation of spinal motoneuron survival by GDNF during development and following injury. Cell Tissue Res. 1996;286:219-223.

29. Li L, Wu W, Lin LF, Lei M, Oppenheim RW, Houenou LJ. Rescue of adult mouse motoneurons from injury-induced cell death by glial cell line-derived neurotrophic factor. Proc Natl Acad Sci USA. 1995;92:9771-9775.

30. Shui C, Scutt AM. Mouse embryo-derived NIH3T3 fibroblasts adopt an osteoblast-like phenotype when treated with $1 \alpha, 25$-dihydroxyvitamin $\mathrm{D}_{3}$ and dexamethasone in vitro. J Cell Physiol. 2002;193:164-172.

31. Satake K, Matsuyama Y, Kamiya M, et al. Up-regulation of glial cell linederived neurotrophic factor (GDNF) following traumatic spinal cord injury. Neuroreport. 2000;11:3877-3881. 